\title{
Looking at the Link between Diet and PCOS Onset and Management
}

\author{
Giusy Porcaro ${ }^{1 *}$, Piero Filati ${ }^{2}$ and Vittorio Unfer $^{3}$ \\ ${ }^{1}$ Department of Obstetrics and Gynecology, University of Perugia, Perugia, Italy \\ ${ }^{2}$ A.G.UN.CO, Obstetrics and Gynecology Center, Rome, Italy \\ ${ }^{3}$ Department of Medical Sciences, IPUS - Institute of Higher Education, Chiasso, Switzerland
}

Received: October 07, 2016; Accepted: November 10, 2016; Published: November 22, 2016

*Corresponding author: Giusy Porcaro, Department of Obstetrics and Gynecology, University of Perugia, Perugia, Italy. E-mail: giusy.porcaro@gmail.com

\begin{abstract}
Polycystic Ovary Syndrome (PCOS) is one of the most common female endocrine disorders which affects about 6-10\% (in some cases $15 \%$ ) of women in reproductive age. It is a chronic, endocrine syndrome that involves the hypothalamus, the pituitary gland, the ovaries, the adrenal gland and the peripheral adipose tissue. Oligoanovulation, hirsutism and infertility are the three main symptoms that characterize it.

Its pathogenesis is not yet entirely clear, but insulin resistance and/or compensatory hyperinsulinemia seem to be central in PCOS onset. Lifestyle management - namely physical activity and diet - is the first line treatment in PCOS. It may help to reduce insulin resistance and restore ovulatory cycles.

Inositol, with its stereo isomers, plays a central role in the functioning of many important metabolic pathways; some of these stereoisomers (mainly myo-inositol and also D-chiro-inositol) are involved in the onset and development of PCOS. This molecule is present in various foods, especially fruits and beans. Today, unfortunately, the refining process of food entails the loss of amino acids, minerals and vitamins. Industrially milled flour is rather bereft of these nutrients because the removal of bran and germ during this step. As a result of such process, refined flour loses one of its main constituents: phytic acid or myo-inositol hexaphosphate. The western diet, typical of industrialized countries, deficient in myo-inositol, increases the risk to develop PCOS in women of childbearing age. Therefore, an improvement of the diet, with appropriate levels of bran, and in some cases the administration of dietary supplements containing inositol (mainly myo-inositol plus D-chiro-inositol at the physiological plasma ratio of 40:1) are highly recommended.
\end{abstract}

Keywords: D-chiro-inositol; Hyperandrogenism; Epimerase; Hyperinsulinemia; Insulin Resistance; Myo-Inositol; Myo-Inositol Hexaphosphate; Phytic Acid; Polycystic Ovary Syndrome;

\section{Introduction}

Polycystic Ovary Syndrome (PCOS) is one of the most common female endocrine disorders. It is estimated that $6-10 \%$ (in some cases 15\%) of women in reproductive age are affected by this syndrome in industrialized countries. It involves the hypothalamus, the pituitary gland, the ovaries, the adrenal gland and the peripheral adipose tissue creating an imbalance associated with three characteristic symptoms: oligoanovulation, hirsutism and infertility. The diagnostic criteria are based on Rotterdam consensus that includes the following three features: oligo-anovulation, clinical or biochemical signs of hyperandrogenism, polycystic-appearing ovaries at ultrasound examination, characterized by the presence of 12 or more follicles with diameter of 2-9 $\mathrm{mm}$ in each ovary, and/or increased ovarian volume ( $>10 \mathrm{ml})$. At least two of them have been fulfilled in order to make the diagnosis.

Despite several studies on PCOS, its patho physiology remains unknown although various etiological factors are suspected to be involved. Particularly insulin resistance and compensatory hyperinsulinemia seem to be central in PCOS onset, in addition to genetic and environmental causes.

\section{Inositol and its therapeutic role}

In the last decades, many scientific studies have focused on inositol(s), which is a polyol existing under nine stereoisomeric forms depending on the spatial orientation of its six hydroxyl groups. Inositol and its derivatives cover a very wide range of physiological activities. The most important molecule is Myo-Inositol (MI), which plays a key function as essential constituent of membrane-incorporated phosphatidylinositol, as well as contributing, per se or conjugated to phosphate, to ion channel permeability, metabolic homeostasis, mRNA export and translation, cytoskeleton remodelling, stress response, just to cite a few [1]. MI and D-chiro-Inositol (DCI), both stereoisomers of inositol, are capable of exerting an insulin-sensitizing effect leading to a reduction of insulin levels in blood, function as insulin second messengers and mediate different insulin actions. MI is converted to an Inositol Phosphoglycan (IPG) insulin second messenger (MI-IPG) which plays a key function in cellular glucose uptake, whereas DCI is converted to an IPG insulin second messenger (DCI-IPG) involved in glycogen synthesis [2]. In the ovary, MI based second messenger is implicated in both glucose 
uptake and FSH signalling whereas DCI-based second messenger is devoted to the insulin-mediated androgen production. John Nestler and his team, provided evidence that the impairment in insulin signalling in PCOS could be the result of a defect in the IPG insulin second messenger pathway [3]. In PCOS women, a deficiency of IPGs in tissues, or altered metabolism of inositol(s) to IPG mediators, could play a role in inducing insulin resistance [3]. It is well known that increasing insulin sensitivity in PCOS patients by means of conventional antidiabetic drugs results in an improvement of ovarian function and decrease of serum androgen concentrations. Several studies have shown that both MI and DCI are able to reduce LH levels, LH/FSH ratio and testosterone levels, to restore spontaneous ovulation and menstrual cycles and to improve cutaneous disorders of hyper androgenism $[4,5]$.

In most tissues almost $99 \%$ of the intracellular inositol pool is constituted by MI. However the concentrations of MI and DCI in fat, muscles and liver are significantly different; such physiological condition is due to the diversified functions that these two stereoisomers play within the tissues. In fact high DCI levels (even if always lower than MI concentration) are generally observed in glycogen storing tissues (fat, liver, muscle), whereas low DCI levels are present in tissues characterized by high consumption of glucose (brain, heart).

\section{The D-chiro-inositol paradox in the ovary}

The conversion of MI to DCI and their different ratios are regulated by an insulin-dependent epimerase according to tissue requirement [6, 7]. As consequence of that, in Type 2 diabetic patients, the reduced tissue insulin sensitivity leads to reduced epimerase activity and hence a decrease in DCI synthesis. However, differently from the other tissues, ovary never shows insulin resistance. Starting from this peculiarity and taking in account a vast array of studies Unfer and co-workers proposed in 2011 the theory called "the DCI paradox in the ovary" which may clarify some features of PCOS $[8,9]$. The authors suggested that hyperinsulinemia leads to an overstimulation of epimerase activity in the ovary of PCOS women resulting in an excessive conversion of MI to DCI, that means, at cellular level, high DCI concentrations with a concomitant depletion of MI. Now it is known that in the normal ovary the predominant stereoisomer of inositol is MI [6]. This evidence, along with studies performed on women undergoing assisted reproductive technologies, demonstrated that MI improves FSH sensitivity. It has led scientists to speculate that MI plays an important role in oocyte quality and development. Consequently the decrease of MI could ultimately be the cause of the poor oocyte quality found in PCOS patients. This evidence was also crucial in order to explain the therapeutic activity of MI in this syndrome [10] and some failures obtained with the administration of DCI alone at elevated doses which determined ovarian toxicity, reducing the ovarian response to FSH and oocyte quality [10]. This could explain why the promising results obtained by Nestler during the first study in patients with PCOS have not been confirmed in the second one, when the dose of DCI given to patients was doubled [3]. The low levels of MI cause a lack of second messengers resulting in chronic anovulation, typical of PCOS. In addition, some studies have allowed to find the physiological concentrations of MI and DCI in plasma of normal women, where these molecules are approximately at the ratio of 40:1 [10-12]. Thus the presumably optimal clinical dosage in order to avoid ovarian toxicity was identified [10, 12]. Of note, the recent Inositol Consensus Conference on the use of MI and DCI in obstetrics, gynecology and fertility [13] formally confirmed the framework suggested by Unfer, adding a new milestone to the promising story of inositol(s).

\section{PCOS and diet}

An uniform treatment for PCOS patients does not exist. Diet therapy is one of the most important and effective management strategies in PCOS. The health benefits of weight loss in overweight and obese women with this syndrome is well established. Weight reduction leads to improve insulin sensitivity and lipid profile; it reduces hyperandrogenism and regulates menstrual cycle [1416].

A diet lower in sugars and higher in complex carbohydrates decreases the postprandial excursion in serum glucose and the excess of insulin secretion in response to dietary load. Low glycemic index carbohydrates are preferable, such as peaches, old-fashioned oatmeal, bran cereals, lentils, sweet potatoes and milk, but also foods with high fiber content and low glycemic index; they promote an increased level of satiety, help to control appetite, decrease hunger and are useful in body weight control. Vegetables contain high quantities of fiber, minerals, vitamins and antioxidants. Non-starchy vegetables are low in calories and carbohydrates. However, the attention for the diet also concerns the quality and characteristics of the food eaten. As well known, over the centuries the eating habits have deeply changed.

\section{Myo-inositol: endogenous synthesis and content in foods and diet}

The refining process to which some foods are subjected, especially flour, caused the reduction in dietary intake of certain substances. MI is the predominant form of inositol(s) that we can find in nature and in our food. It is synthesized by human body from glucose-6-phosphate. Liver and kidneys are the key organs for its endogenous synthesis, however such biosynthesis does not seem to be able in providing adequate amounts of MI for a good health, at least in some groups of subjects. Human diet from animal and plant sources can contain MI in its free form, as inositol-containing phospholipid (phosphoinositides) or as myo-inositol-1,2,3,4,5,6-hexakis dihydrogen phosphate, also called phytic acid (IP6). Phytate, the salt of phytic acid, is widely distributed in the plant kingdom. It serves as a storage form of phosphorous and minerals and contains about $75 \%$ of total phosphorous of the kernels, serving biologically for birth and bud development. The greatest amounts of MI in its different forms are found in fresh fruits and vegetables, and in all foods containing seeds (beans, grains and nuts). Especially high phytic acid contents are found in almonds, walnuts and Brazil nuts; bran and oats contain more MI than other sources. Among vegetables, the highest contents are found in the beans and peas, instead the 
poorest in leafy vegetables. Among the fruits, melon and citrus fruits (with the exception of lemons) have extraordinarily high contents of MI [17].

MI can be released from IP6 in the gut of monogastric animals by phytate-degrading enzymes, called phytases, which are found in plants, microorganisms and animal tissues. Much of the ingested inositol hexaphosphate is hydrolysed to inositol available to be used by the cell. As we know, through the decades our diet has changed considerably. The refining process of flour has brought to the depletion of many substances, among them MI has to be included also for a reason that concerns it specifically.

\section{Scaling down the antinutrient activity of phytates}

Phytic acid forms complexes as mixed salts phytates, with many essential bi- and trivalent metal ions, in foods as well as in the intestine. It shows the feature to easily and severally bind to calcium, iron, magnesium, manganese, zinc, but potentially to other ions and proteins. The chelating properties of phytic acid may, therefore, have an effect on the bioavailability of minerals. This is the reason why it is considered an antinutrient compound. Due to this feature of phytic acid, there has been a lot of concern about the effect of an unrefined highfiber diet on health. Consequently many researchers have been carried out to remove phytate from food, to avoid deficiencies of essential minerals. Reducing the dietary intake of phytic acid, MI intake was also decreased. Therefore dietary changes, due to the reduced consumption of this component, may have contributed to facilitate the onset and the development of PCOS in the population of industrialized countries in consideration of the central role exerted by MI in the functioning of important metabolic pathways, involved in PCOS. More recently it has been suggested that phytic acid is not the component that is solely responsible for the decreased mineral availability, as dietary fiber itself also might be of importance $[18,19]$. On the other hand in the last decades also beneficial properties of phytate have been observed. It prevents renal stone formation, reduces starch digestion along with slowing down of the glycemic index of foods, has positive effects on blood glucose and blood cholesterol [20-23]. Furthermore, it exerts antioxidant [24] and anticancer activities [25]. These findings have revived speculation about the role of phytate and other inositol phosphates in human nutrition and for human health.

\section{Phytate absorption}

It is important to understand what occurs to phytate in the gut and how phytate and its degradation products can be absorbed. The human beings do not synthesize the phytase enzyme, but nature has given us the same chance to digest phytate and phytic acid. First, exploiting this enzyme present in the seeds: the acid and hot stomach environment, in fact, actives large part of phytases. Some studies [26] have shown that a lot of phytic acid can be neutralized already in the stomach and make free those minerals linked to it. IP6, partially degraded, is more easily digested then in the intestine. The amount remained intact passes through the small intestine and may undergo further dephosphorylation in the colon by the intestinal bacteria. However a clarification should be made. This process of degradation and assimilation of IP6 occurs in ideal conditions: primarily when the consumed seeds have not undergone an excessive manipulation and secondly through an efficient digestive system, possibly not weakened by a poor or improper diet. Potentially a broad range of inositol phosphates may be consumed, eating unprocessed food. It has been estimated that the daily intake of phytate and other inositol phosphates varies from 0.180 to $4.569 \mathrm{~g}$ [27]. Obviously significant differences may exist between developing and industrialised countries, urban and rural areas, females and males, young and old, omnivores and vegetarians, however specific studies are still lacking [17]. This variability derives from differences in phytate content of cultivated plant foods, different contents in plant foods in the daily diet, different amounts of foods consumed and different processing and preparation of vegetables, legumes, pulses, cereals and whole grain products. Strong variations between the mean phytate intake in rural population (greater intake of phytic acid) and urban population (lower intake of phytic acid) are evident in the Republic of China [28]. These data reflect the changing of dietary habits from traditional to more Westernized type diets in cities and metropolises. In the industrialized countries, most plant foods, such as whole grain products - the main sources of dietary phytate intake - are processed or heat treated either during food production or preparation, in such way phytases are inactivated or removed to a large extent $[17,29]$. This is another dangerous depletion which adds to the reduced content of phytate in refined foods. It means that in humans consuming Western style diets with low phytase activity, phytate degradation in the stomach and the small intestine by food phytases is very limited and consequently the amount of MI absorbed with the diet nowadays is even more reduced.

\section{Balanced diet for improving phytate digestion and dietary supplements}

In fact, under non-varied and non-balanced diet, phytate may affect the bioavailability and in consequence the status of some minerals (iron, zinc and calcium) [30]. It should be stressed that in many countries whole grain cereals and legumes are among the most important food sources for minerals and trace elements but also contain high amounts of phytate and polyphenols. Adequate strategies to prevent deficiencies of these essential elements adjusted to the specific situation are required, in order to preserve the enormous advantages of the consumption of whole grains, legumes and oil seeds decreasing its negative effects. It is advisable, therefore, a varied diet. In fact the minerals from other foods are not chelated by acid phytic during their passage in the intestine. It is important a proper intake of fibers in the diet. Especially the soluble (found mostly in fruits and vegetables) fermenting in the colon by the action of healthy intestinal bacterial communities produce short-chain fatty acids that can easily bind to the mineral ions making them assimilable and eliminating the negative effect of IP6. If we take into account the leavening process of the dough for bread, pizzas and cakes, this speech becomes more complex. The hydrolysis of phytate occurs by the activation of the endogenous phytase of 
flour. In the case of bread made with sourdough leavening it is more effective than one made with yeast because of the longer times required. In a well-leavened dough up to $100 \%$ of phytic acid is hydrolyzed. But to allow proper hydrolysis of phytic acid the leavening should be long, not that quickly induced by industrial yeasts, used by most of the industry, and unfortunately also by many bakers. It is understood, therefore, as the inhibition of the intestinal metal absorption, however, can be counteracted by many food compounds such as organic acids and complexing agents, ascorbic acid, food fermentation products. Milling of cereals, in which phytate is located in the outer layer of the seed, can cause up to $90 \%$ reduction of phytate. In corn where phytate is mainly located in the germ, removing of this part of the grain will effectively result in strong reduction of it. Mechanical separation of the phytate containing compartments of the seeds, however, will also lead to a loss of nutrients and valuable bioactive molecules. All this to prevent its chelating action that in the light of recent studies appears to be very minimal and which can further be reduced with small dietary precautions.

Whether a balanced diet and a correct food processing can facilitate the right intake of phytates, however in many case a satisfying condition cannot be achieved in specific population sectors by these means. It is well known that, in agreement with the Rotterdam criteria, four different "discrete" phenotypes, or subsets, can be identified in PCOS women [31-33]. Therefore, we may allocate them in the following groups of subjects affected by two or three clinical and endocrine anomalies:

1. chronic ovulatory disorder (OD) + hyperandrogenism (HA) + polycystic ovaries (PCO),

2. chronic ovulatory disorder (OD) + hyperandrogenism (HA),

3. hyperandrogenism (HA) + polycystic ovaries (PCO),

4. chronic ovulatory disorder (OD) + polycystic ovaries (PCO)

The most common subset is the phenotype 1, with $60,2 \%$, whereas the other groups showed respectively these frequencies: group 2 with $16,1 \%$, 3 with 18,3\%, and 4 with 5,4\%. [33]. In many of these subjects it may be necessary associating to a correct diet also the intake of dietary supplements containing inositol. Recent researches and studies have highlighted the relevance of the supplementation with MI plus DCI at the physiological plasma ratio of 40:1 in PCOS therapy $[7,10]$. This kind of treatment allows to restore the correct balance between these two molecules in the body.

\section{Conclusion}

Phytic acid is one of the most fascinating bioactive food compounds and is widely distributed in plant foods. It is an important source of MI for our organism. Due to its molecular structure, phytic acid shows a high affinity to polyvalent cations and interferes in their intestinal absorption.

In countries where the diet is unbalanced this may lead to serious deficiencies. However, with a well-balanced nutrition this seems to be a less significant problem.
These considerations let us to think that PCOS may be a characteristic syndrome in the industrialized countries. The modern food processing allows us to have attractive and highly refined foods that have lost some of their essential nutritional characteristics.

In industrialised countries the beneficial effect of phytic acid on the blood glucose and blood cholesterol levels, its anticancer and antioxidant effect should advise its dietary intake.

Aspects highlighted on the pathogenesis of PCOS and the role of MI as insulin-sensitizing agent show that the eating habits of Westernized populations and the quality of the food we eat expose us to a low intake of phytic acid.

We can conclude that a diet rich in foods containing phytic acid would help to improve the quality of life of patients with PCOS, reducing probably its incidence in the population and its complications in the short and long term. The changes in lifestyle and eating habits still represent the first-line treatment in PCOS. Furthermore, we cannot forget the therapeutic efficacy of a dietary supplement containing MI and DCI, at their physiological plasma ratio of 40:1, in restoring the correct balance between these two molecules in the body, mainly in cases such as PCOS.

\section{Competing Interests}

Vittorio Unfer is employed at Lo.Li.Pharma SRL, Rome. The other authors declare that they have no conflict of interests regarding the publication of this paper.

\section{References}

1. Bizzarri M, Fuso A, Dinicola S, Cucina A, Bevilacqua A. Pharmacodynamics and pharmacokinetics of inositol(s) in health and disease. Expert Opin Drug Metab Toxicol. 2016;12(10):11811196. doi: $10.1080 / 17425255.2016 .1206887$

2. Larner J, Huang LC, Tang G, S Suzuki, CFW Schwartz, G Romero, et al. Insulin mediators: structure and formation. Cold Spring Harb Symp Quant Biol. 1988;53:965-971. doi:10.1101/SQB.1988.053.01.111

3. Nestler JE, Unfer V. Reflections on inositol(s) for PCOS therapy: steps toward success. Gynecol Endocrinol. 2015;31(7):501-505. doi: 10.3109/09513590.2015.1054802

4. Nestler JE, Jakubowicz DJ, Reamer P, Gunn RD, Allan G. Ovulatory and metabolic effects of D-chiro-inositol in the polycystic ovary syndrome. N Engl J Med. 1999;340(17):1314-1320. doi: $10.3109 / 09513590.2015 .1054802$

5. Zacchè MM, Caputo L, Filippis S, Zacchè G, Dindelli M, Ferrari A. Efficacy of myo-inositol in the treatment of cutaneous disorders in young women with polycystic ovary syndrome. Gynecol Endocrinol. 2009;25(8):508-513. doi: 10.1080/09513590903015544

6. Heimark D, McAllister J, Larner J. Decreased myo-inositol to chiroinositol $(\mathrm{M} / \mathrm{C})$ ratios and increased M/C epimerase activity in PCOS theca cells demonstrate increased insulin sensitivity compared to controls. Endocr J. 2014;61(2):111-117.

7. Dinicola S, Chiu TT, Unfer V, Carlomagno G, Bizzarri M. The rationale of the myo-inositol and D-chiro-inositol combined treatment for polycystic ovary syndrome. J Clin Pharmacol. 2014;54(10):10791092. doi: 10.1002/jcph.362.

8. Carlomagno G, Unfer V, Roseff S. The D-chiro-inositol paradox in the ovary. Fertil Steril. 2011;95(8):2515-2516. doi: 10.1016/j. fertnstert.2011.05.027 
9. Unfer V, Carlomagno G, Papaleo E, Vailati S, Candiani M, Baillargeon JP. Hyperinsulinemia Alters Myoinositol to d-chiroinositol Ratio in the Follicular Fluid of Patients With PCOS. Reprod Sci. 2014;21(7):854858.

10.Unfer V, Porcaro G. Updates on the myo-inositol plus D-chiro-inositol combined therapy in polycystic ovary syndrome. Expert Rev Clin Pharmacol.2014;7(5):623-631. doi: 10.1586/17512433.2014.925795

11.De Grazia S, Carlomagno G, Unfer V, Cavalli P. Myo-inositol soft gel capsules may prevent the risk of coffee-induced neural tube defects. Expert Opin Drug Deliv. 2012;9(9):1033-1039. doi: 10.1517/17425247.2012.701616

12. Facchinetti F, Dante G, Neri I. The Ratio of MI to DCI and Its Impact in the Treatment of Polycystic Ovary Syndrome: Experimental and Literature Evidences. Front Gynecol Endocrinol. ISGE Series. 2015;3:103-109.

13. Bevilacqua, G. Carlomagno, S. Gerli, Montanino Oliva M, Devroey $\mathrm{P}$, Lanzone $\mathrm{A}$, et al. Results from the international consensus conference on myo-inositol and D-chiro-inositol in obstetrics and gynecology - assisted reproduction technology. Gynecol Endocrinol. 2015;31(6):441-446. doi: 10.3109/09513590.2015.1006616

14.Moran LJ, Noakes M, Clifton PM, Tomlinson L, Galletly C, Norman RJ. Dietary composition in restoring reproductive and metabolic physiology in overweight women with polycystic ovary syndrome. J Clin Endocrinol Metab. 2003;88(2):812-819.

15.Crosignani PG, Colombo M, Vegetti W, Somigliana E, Gessati A, Ragni G. Overweight and obese anovulatory patients with polycystic ovaries: parallel improvements in anthropometric indices, ovarian physiology and fertility rate induced by diet. Hum Reprod. 2003;18(9):19281932.

16. Herriot AM, Whitcroft S, Jeanes Y. An retrospective audit of patients with polycystic ovary syndrome: the effects of a reduced glycaemic load diet. J Hum Nutr Diet. 2008;21(4):337-345. doi: 10.1111/j.1365277X.2008.00890.x.

17.Schlemmer U, Frølich W, Prieto RM, Grases F. Phytate in foods and significance for humans: food sources, intake, processing bioavailability, protective role and analysis. Mol Nutr Food Res. 2009;53 Suppl 2:S330-375. doi: 10.1002/mnfr.200900099.

18. Frølich W. Chelating properties of dietary fiber and phytate. The role for mineral availability. Adv Exp Med Biol. 1990;270:83-93.

19. Péneau S, Dauchet L, Vergnaud AC, Estaquio C, Kesse-Guyot E, Bertrais $\mathrm{S}$, et al. Relationship between iron status and dietary fruit and vegetables based on their vitamin $\mathrm{C}$ and fiber content. Am J Clin Nutr. 2008;87(5):1298-1305.
20.Grases F, Costa-Bauzá A. Phytate (IP6) is a powerful agent for preventing calcifications in biological fluids: usefulness in renal lithiasis treatment. Anticancer Res. 1999;19(5A):3717-3722.

21. Thompson LU, Button CL, Jenkins DJ. Phytic acid and calcium affect the in vitro rate of navy bean starch digestion and blood glucose response in humans. Am J Clin Nutr. 1987;46(3):467-473.

22. Lee SH, Park HJ, Chung H. K. Choa S-Y, Choa S-M, Lillehoj HS. Dietary phytic acid lowers the blood glucose level in diabetic KK mice. Nutr Res. 2006;26(9):474-479.

23. Jariwalla RJ, Sabin R, Lawson S, Herman ZS. Lowering of serum cholesterol and triglycerides and modulation of divalent cations by dietary phytate. J Appl Nutr. 1990; 42(1):18-28.

24. Graf E, Empson KL, Eaton JW. Phytic acid. A natural antioxidant. J Biol Chem. 1987;262(24):11647-11650.

25. Shamsuddin AM. Inositol phosphates have novel anticancer function. J Nutr. 1995;125(3 Suppl):725S-732S.

26. Grases F, Simonet BM, Vucenik I, Prieto RM, Costa-Bauzá A, March JG, et al. Absorption and excretion of orally administered inositol hexaphosphate (IP(6) or phytate) in humans. Biofactors. 2001;15(1):53-61.

27. Reddy NR, Sathe SK. Food Phytates: CRC Press, Boca Raton, London, New York, Washington, 2002;25-51.

28. Cheng Y, Dibley MJ, Zhang X, Zeng L, Yan H. Assessment of dietary intake among pregnant women in a rural area of western China. BMC Public Health. 2009;9:222. doi: 10.1186/1471-2458-9-222.

29. Gupta RK, Gangoliya SS, Singh NK. Reduction of phytic acid and enhancement of bioavailable micronutrients in food grains. J Food Sci Technol. 2015;52(2):676-684. doi: 10.1007/s13197-013-0978-y

30.Zimmermann MB, Hurrell RF. Nutritional iron deficiency. Lancet. 2007;370(9586):511-520.

31. Moran L, Teede H. Metabolic features of the reproductive phenotypes of polycystic ovary syndrome. Hum Reprod Update. 2009;15(4):477488. doi: 10.1093/humupd/dmp008.

32. Welt CK, Gudmundsson JA, Arason G, Adams J, Palsdottir H, Gudlaugsdottir G, et al. Characterizing discrete subsets of polycystic ovary syndrome as defined by the Rotterdam criteria: the impact of weight on phenotype and metabolic features. J Clin Endocrinol Metab. 2006;91(12):4842-4848.

33. Głuszak O, Stopińska-Głuszak U, Glinicki P, Kapuścińska R, Snochowska H, Zgliczyński W, et al. Phenotype and Metabolic Disorders in Polycystic Ovary Syndrome, ISRN Endocrinol. 2012;2012:569862. doi: $10.5402 / 2012 / 569862$. 